\title{
Histone Deacetylase Inhibitors Decrease Cocaine But Not Sucrose Self-Administration in Rats
}

\author{
Pascal Romieu, ${ }^{1,4 *}$ Lionel Host, ${ }^{1,4 *}$ Serge Gobaille, ${ }^{2,4}$ Guy Sandner, ${ }^{3,4}$ Dominique Aunis, ${ }^{1,4}$ and Jean Zwiller ${ }^{1,4}$ \\ ${ }^{1}$ Inserm, U575, Centre de Neurochimie, 67084 Strasbourg, France, ${ }^{2}$ Institut Fédératif de Recherche 37 Neurosciences, 67085 Strasbourg, France, ${ }^{3}$ Inserm, \\ U666, 67085 Strasbourg, France, and ${ }^{4}$ Université Louis Pasteur, 67084 Strasbourg, France
}

\begin{abstract}
Regulation of gene expression is known to contribute to the long-term adaptations taking place in response to drugs of abuse. Recent studies highlighted the regulation of gene transcription in neurons by chromatin remodeling, a process in which posttranslational modifications of histones play a major role. To test the involvement of epigenetic regulation on drug-reinforcing properties, we submitted rats to the cocaine operant self-administration paradigm. Using the fixed ratio 1 schedule, we found that the histone deacetylase (HDAC) inhibitors trichostatin A and phenylbutyrate dose-dependently reduced cocaine self-administration. Under the progressive ratio schedule, both trichostatin A and depudecin significantly reduced the breaking point, indicating that HDAC inhibition attenuated the motivation of rats for cocaine. Conversely, HDAC inhibition did not decrease self-administration for the natural reinforcer sucrose. This observation was correlated with measurements of HDAC activity in the frontal cortex, which was inhibited in response to cocaine, but not to sucrose self-administration. Control experiments showed that the decrease in the motivation for the drug was not attributable to a general motivational dysfunction because trichostatin A had no adverse effect on locomotion during the habituation session or on cocaine-induced hyperlocomotion. It was not attributable to anhedonia because the inhibitor had no effect on the sucrose preference test. In contrast, trichostatin A completely blocked the cocaine-induced behavioral sensitization. Together, the data show that epigenetic regulation of gene transcription in adult brain is able to influence a motivated behavior and suggest that HDAC inhibition may counteract the neural sensitization leading to drug dependence.
\end{abstract}

Key words: cocaine; sucrose; epigenetic; histone modification; self-administration; addiction

\section{Introduction}

Drug dependence is currently viewed as a chronic brain disease characterized primarily by a compulsive drug-seeking and drugtaking behavior. The development of dependence to drugs of abuse occurs over time and requires cellular adaptations. One area of intensive research aimed at identifying the long-term adaptations is the regulation of changes in gene expression evoked by the drugs (Nestler, 2001). For instance, cocaine administration has been shown to elicit induction in neurons of several immediate early genes that encode transcription factors (Hope et al., 1992; Moratalla et al., 1992; Bhat and Baraban, 1993; Humblot et al., 1998; Jouvert et al., 2002). Although much of the work has focused on these factors often considered to be molecular switches that coordinate changes in gene expression underlying neuronal plasticity, the molecular mechanisms which control expression of genes, referred to as epigenetic mechanisms, have been under appreciated in the nervous system. Recent studies highlighted the importance in neurons of chromatin remodeling

\footnotetext{
Received Jan. 28, 2008; revised July 23, 2008; accepted July 23, 2008.

We gratefully acknowledge resources provided by the Institut de Recherches Scientifiques sur les Boissons and the support of the Société Française d'Alcoologie.

*P.R. and L.H. contributed equally to this work.

Correspondence should be addressed to Pascal Romieu, Inserm U575, Centre de Neurochimie, 5 rue Blaise Pascal, 67084 Strasbourg Cedex, France. E-mail: romieu@neurochem.u-strasbg.fr. DOI:10.1523/JNEUROSCI.0379-08.2008

Copyright $\odot 2008$ Society for Neuroscience $\quad 0270-6474 / 08 / 289342-07 \$ 15.00 / 0$
}

in controlling gene transcription and revealed that complex mechanisms regulate the accessibility of genes to the transcriptional machinery (Colvis et al., 2005). Access to DNA in the nucleosome is achieved via complex associations of proteins in which posttranslational modifications of histones play a major role. In general, increased histone acetylation is associated with DNA relaxation and elevated transcriptional activity, whereas decreased acetylation brought about by histone deacetylases (HDACs) and methyl-CpG-binding proteins such as MeCP2 results in tighter DNA coiling and gene silencing (López-Rodas et al., 1993; Jenuwein and Allis, 2001; Turner, 2002).

We demonstrated that repeated injections of cocaine as well as of the antidepressant fluoxetine regulate chromatin remodeling by inducing the methyl-CpG-binding proteins $\mathrm{MeCP} 2$ and MBD1 in rat striatum and frontal cortex (Cassel et al., 2006). The data suggest that an epigenetic regulation mechanism of gene transcription could be involved in the behavioral long-term effects elicited by cocaine. To test the hypothesis, we treated rats with HDAC inhibitors and submitted them to the intravenous cocaine operant self-administration paradigm. The effects of chromatin remodeling on cocaine-taking behavior were compared with those observed during self-administration of sucrose, a natural reinforcer. We used the fixed ratio (FR) schedule, in which rats have to produce a fixed number of nose-pokes (NPs) into a hole to obtain a cocaine or sucrose infusion. Although this experiment measures the reinforcing properties of the com- 
pounds, the evaluation of the motivation of animals for a given compound is assessed by the progressive ratio (PR) schedule, in which the self-administration task is rendered more difficult, because the number of NPs required for an infusion increases progressively (Depoortere et al., 1993; Arnold and Roberts, 1997).

\section{Materials and Methods \\ Animals}

Male Wistar rats (Janvier), weighing 160-180 g, were housed in standard home cages (five rats per cage), under an inverted $12 \mathrm{~h}$ light/dark cycle (lights on at 7:00 P.M.), with water available from two bottles. Animals had ad libitum access to food and water, unless indicated otherwise. After surgery for catheter implantation into the jugular vein, animals were housed individually so that acoustic, olfactive, and visual social contacts remained possible. They were allowed to recover for 5-7 d before the beginning of behavioral tests which were conducted during the dark period. Each of the specific behavioral tests was performed using distinct groups of rats. All procedures involving animal care were conducted in compliance with current laws and policies (Council directive 87848, Service Vétérinaire de la Santé et de la Protection Animales).

\section{Pharmacological treatment}

Cocaine hydrochloride (Sigma-Aldrich) solution was adjusted with $0.9 \%$ $\mathrm{NaCl}$ to infuse an intravenous dose of $0.33 \mathrm{mg} / \mathrm{kg} /$ injection for selfadministration and $1 \mathrm{mg} / \mathrm{kg}$ for locomotor activity. Trichostatin A (TsA; Sigma-Aldrich), sodium 4-phenylbutyrate (Fluka, Sigma-Alrich), depudecin (Sigma-Aldrich) or 10\% DMSO vehicle solution were administered 30 min before the beginning of the self-administration session. TsA and phenylbutyrate were injected intravenously, whereas depudecin was administered intracerebroventricularly.

\section{Surgery}

Intravenous catheterization procedure was performed as described previously (Caine et al., 1993). Rats were anesthetized by the intraperitoneal injection $(1 \mathrm{ml} / \mathrm{kg})$ of a 5:1 (v/v) mixture containing $0.1 \mathrm{~g} / \mathrm{ml}$ ketamine (Imalgene 1000) and 2\% xylazine (Rompun; Centravet), before surgical implantation of a chronic indwelling catheter in the right jugular vein. The catheter, which consisted of SILASTIC tubing $12 \mathrm{~cm}$ long [ $0.3 \mathrm{~mm}$ inner diameter (i.d.) $\times 0.63 \mathrm{~mm}$ outer diamter (o.d.)] (SILASTIC; Plastics One), was fitted to a 22 gauge guide cannula (Plastics One) that was bent at a right angle and then embedded in dental cement on a circular $2.5 \mathrm{~cm}$ mercylene mesh base. A discrete incision was performed onto the jugular vein; the heparinized catheter was immediately inserted $3.6 \mathrm{~cm}$ into the vein and anchored with suture. Catheters were flushed daily with $150 \mu \mathrm{l}$ of a solution mixture containing $300 \mathrm{U} / \mathrm{ml}$ heparin and $0.1 \mathrm{~g} / \mathrm{ml}$ ampicillin to prevent clotting and infection.

\section{Cocaine operant self-administration}

Apparatus. Studies were conducted in dark operant chambers $(30 \times 30 \times$ $30 \mathrm{~cm}$ ) located in a sound-attenuated room. A single channel fluid swivel (Instech Laboratories) was mounted on a balanced arm above each chamber. A computer-driven syringe pump (Imetronic) activated a $5 \mathrm{ml}$ syringe and pushed fluid into SILASTIC tubing $(0.63 \mathrm{~mm}$ i.d. $\times 1.19 \mathrm{~mm}$ o.d.) connected to the rat through its externalized 22 gauge guide cannula. Each chamber was equipped with two $3 \mathrm{~cm}$-diameter holes on the same wall, located $4 \mathrm{~cm}$ above the floor; one was selected as the active hole for delivering the reinforcer and the other as the inactive hole. They were counterbalanced between right and left position in the various groups of rats. Disruption of an infrared photobeam in each hole was detected using a digital input card (DIO-24; National Instruments) and home made LabView software (National Instruments). NPs into both holes were recorded. NPs into the inactive hole had no programmed consequence. When the number of NPs into the active hole was reached, a 40 $\mu$ l cocaine solution was delivered $>2$ s under the control of the computer. A stimulus light, located $20 \mathrm{~cm}$ above the active hole, was paired contingently with the delivery of cocaine and persisted during a $40 \mathrm{~s}$ time-out period.

Drug self-administration procedure. For cocaine self-administration procedure (LeSage et al., 1999), rats were first submitted to an FR1 sched- ule of reinforcement during daily $2 \mathrm{~h}$ sessions for $7 \mathrm{~d}$. In this schedule, each NP into the active hole triggered an intravenous infusion of cocaine solution. No cutoff was applied concerning the number of self-infusions the rat was able to perform during the session. The day after the seventh FR1 session, rats were submitted to a PR schedule, in which the NPs required to earn an injection escalated according to the following exponential equation (Richardson and Roberts, 1996), where $n$ is the rank number of successive injections: $\mathrm{NP}_{(n)}=\left[5 \cdot e^{0.2 \cdot n}\right]-5$.

The PR schedule was performed for 7 consecutive daily sessions. Each session lasted for $5 \mathrm{~h}$ or until animals did not achieve the ratio for delivery of an injection within $1 \mathrm{~h}$. The breaking point to extinguish selfadministration behavior was determined in each animal.

\section{Sucrose operant self-administration}

Apparatus. Studies were conducted in the same chambers than those used for cocaine self-administration. A window $(4 \times 4 \mathrm{~cm}$; bottom at $4 \mathrm{~cm}$ above the floor) located on the wall adjacent to the active hole allowed the rat to drink a sucrose solution from a container placed outside the chamber. When the number of required NPs into the active hole was achieved, $300 \mu \mathrm{l}$ of a $10 \%$ sucrose solution were delivered into the container over a $3 \mathrm{~s}$ period through a syringe controlled by a computer-driven pump.

Sucrose self-administration procedure. Rats were submitted to FR1 and PR sessions, as described for cocaine self-administration. Two days after surgery for catheter implantation, rats were submitted to a $48 \mathrm{~h}$ period of exposure to $4 \%$ sucrose solution to habituate to it. Tap water was then substituted for the sucrose solution. Seven days after surgery, rats were submitted to water deprivation for $20 \mathrm{~h}$ and were then placed for $1 \mathrm{~h}$ in the operant chamber with the container holding $2 \mathrm{ml}$ of a $10 \%$ sucrose solution. At the end of the shaping session, rats were returned to home cages and were free to drink tap water for $4 \mathrm{~h}$, after which the procedure was repeated once. An operant conditioning session, without any treatment, began under FR1 the day after the second shaping session and allowed to split the rats into two balanced groups. Pharmacological treatment, followed by operant conditioning session, began the next day.

\section{Sucrose preference test}

Three weeks after their arrival in the laboratory, rats were submitted to a $48 \mathrm{~h}$ period during which a $1 \%$ sucrose solution was the only fluid available. Fluid was available from two bottles throughout the experiment. Rats were then housed individually, with the sucrose solution being available for another day, followed by a $3 \mathrm{~d}$ period during which water was substituted for sucrose. They were pretested for sucrose preference on the following day. Rats were placed individually in test cages, first with no access to fluid for $3 \mathrm{~h}$, and then with access to the two bottles, one containing tap water and the other a $1 \%$ sucrose solution. Fluid intake was measured by weighing the bottles after a $60 \mathrm{~min}$ period in the test cage and percentage of sucrose preference was calculated (volume of sucrose intake $\times 100 /$ total fluid intake). Rats were returned to their home cage with tap water available ad libitum, and $2 \mathrm{~d}$ later they underwent surgery for catheter implantation as described above. After a $3 \mathrm{~d}$ recovery period, they were injected daily for $3 \mathrm{~d}$ with TsA $(0.3 \mathrm{mg} / \mathrm{kg}$, i.v. $)$ or vehicle. On the following day, animals were tested again for sucrose preference $30 \mathrm{~min}$ after the fourth TsA injection.

\section{Locomotor activity}

To measure spontaneous locomotor activity, rats were placed individually in Plexiglas cages (base $30 \times 45 \mathrm{~cm}$; height $35 \mathrm{~cm}$ ). The floor was covered with sawdust and a passive infrared detector (IRP124; Talco) was placed above the cage behind a Fresnel lens which divides the cage into sectors. The detector was controlled by a home made Labview Software (National Instruments). It recorded the number of times the rat crossed limits of a beam every $5 \mathrm{~min}$. Rats were treated daily with TsA $(0.3 \mathrm{mg} / \mathrm{kg})$ or vehicle for $4 \mathrm{~d}$. Thirty minutes after the last injection, they were placed into the test cage (the cage being a new environment for them), and locomotor activity was recorded for $2 \mathrm{~h}$. For measurement of cocaineinduced hyperlocomotor activity, rats were given an intravenous cocaine injection after a $90 \mathrm{~min}$ habituation session in the test chamber, and locomotor activity was monitored over the next $90 \mathrm{~min}$ period. This treatment was repeated each day during $5 \mathrm{~d}$, and locomotor activity was recorded again after a $6 \mathrm{~d}$ drug-free period. 


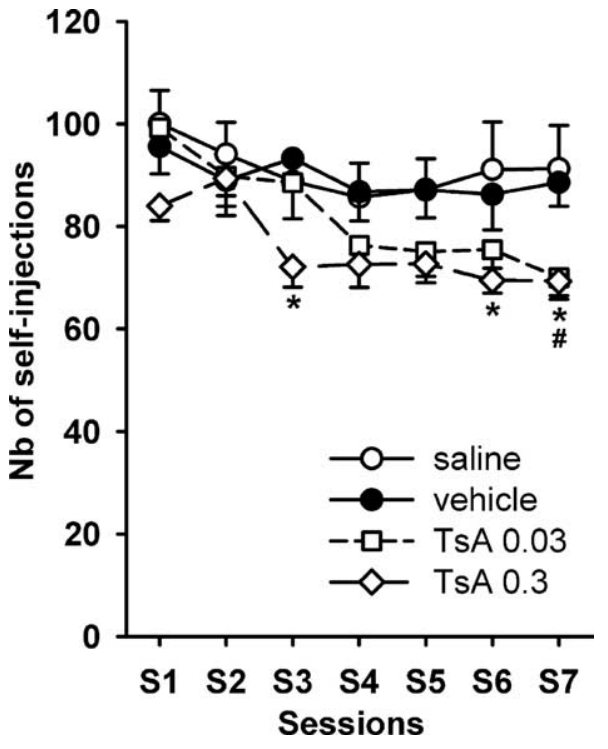

Figure 1. Effect of TsA on cocaine self-administration $(0.33 \mathrm{mg} / \mathrm{kg} /$ infusion) under the FR1 schedule of reinforcement. TsA or vehicle (10\% DMS0) was administered intravenously 30 min before the beginning of each cocaine self-administration session. The number of self-injections reached during $2 \mathrm{~h}$ daily sessions is expressed as mean $\pm \operatorname{SEM}$. Control $(n=10)$, vehicletreated $(n=12), 0.03 \mathrm{mg} / \mathrm{kg}$ TsA-treated $(n=13)$, and $0.3 \mathrm{mg} / \mathrm{kg}$ TsA-treated $(n=10)$ groups of rats were studied. ${ }^{*} p<0.05$, comparison between vehicle-treated and $0.3 \mathrm{mg} / \mathrm{kg}$ TsA-treated groups; ${ }^{*} p<0.05$, comparison between vehicle-treated and $0.03 \mathrm{mg} / \mathrm{kg}$ TsAtreated groups (ANOVA and Student-Newman-Keuls post hoc test). Nb, Number.

\section{HDAC assay}

Total nuclear HDAC activity was measured by using the Fluor de Lys assay system (BIOMOL). After treatment, rat prefrontal cortex (FCx) and nucleus accumbens (NAc) were dissected and homogenized in 20 mм HEPES buffer, $\mathrm{pH} 7.9$, containing $0.1 \mathrm{~m} \mathrm{KCl}, 0.2 \mathrm{~mm}$ EDTA, $0.5 \mathrm{~mm}$ dithiothreitol, $0.5 \mathrm{~mm}$ phenylmethylsulfonyl fluoride, and $20 \%$ glycerol. The homogenate was centrifuged at $1000 \times g$ for $20 \mathrm{~min}$ at $4^{\circ} \mathrm{C}$, and HDAC activity was assayed in the pellet resuspended in the same buffer. Assays were conducted at $30^{\circ} \mathrm{C}$ for $20 \mathrm{~min}$ in a reaction mixture $(50 \mu \mathrm{l}$ total volume) containing $50 \mathrm{~mm}$ Tris/Cl buffer, $\mathrm{pH}$ 8.0, $137 \mathrm{~mm} \mathrm{NaCl}, 2.7$ $\mathrm{mm} \mathrm{KCl}, 1 \mathrm{~mm} \mathrm{MgCl}_{2}, 250 \mu \mathrm{M}$ acetylated lysine side chain substrate, and $20 \mu \mathrm{g}$ of protein extract. Enzymatic reaction was stopped by the addition of $50 \mu \mathrm{l}$ of a $2 \mu \mathrm{M}$ TsA solution and fluorescence was generated from deacetylated substrate by the addition of a fluorescence developer. After $15 \mathrm{~min}$, the fluorescence produced was measured in a 96-well fluorescence plate reader (MicroLumat Plus LB; excitation, $380 \mathrm{~nm}$; emission, $460 \mathrm{~nm}$ ). Deacetylase activity was expressed as arbitrary fluorescence units per min per mg of protein.

\section{Statistical analysis}

Results are expressed as means \pm SEM. Effects of the HDAC inhibitors during the totality of the FR sessions and for the three last PR sessions, as well as on the locomotor activity during cocaine-induced sensitization, were analyzed by two-way ANOVA (treatment and session as factors of variation) between groups of rats. Effects on HDAC activity measurements and on locomotor activity on the first day of sensitization were analyzed by one-way ANOVA (treatment as factor of variation). Student-Newman-Keuls post hoc tests were performed when required, and significance was set at $p \leq 0.05$.

\section{Results}

\section{Effect of HDAC inhibitors on cocaine self-administration}

When trained during daily $2 \mathrm{~h}$ sessions of cocaine selfadministration under the FR1 schedule of reinforcement, salinetreated rats and rats treated with 10\% DMSO (vehicle) displayed a stable level of cocaine intake after 3 sessions (Fig. 1). Using a cocaine dosage of $0.33 \mathrm{mg} / \mathrm{kg} /$ infusion, rats self-administered $\sim 90$ injections. Number of cocaine self-infusions was very similar whether animals were injected intravenously with saline or vehicle, showing that the vehicle solution did not affect the selfadministration behavior. It is noteworthy that rats that selfadministered cocaine after being injected with saline or vehicle directed $>80 \%$ of the NPs onto the active hole (data not shown). Daily intravenous administration of TsA 30 min before the beginning of the self-administration session resulted in a significant reduction in cocaine intake $\left(F_{(3,314)}=11.4 ; p<0.001\right)$. The lower dose of $0.03 \mathrm{mg} / \mathrm{kg}$ TsA produced a statistically significant decrease in cocaine self-injections only in session 7 (Fig. 1). The higher dose of $0.3 \mathrm{mg} / \mathrm{kg}$ TsA produced a statistically significant effect as early as in session 3, for which a $23 \%$ reduction in selfinjections was observed, but it did not reach statistical significance on the subsequent sessions 4 and 5. The statistical significance was met for sessions 6 and 7. When the animals, once showing reduced cocaine self-administration, were subjected to further FR1 sessions but without previous TsA injection, a decrease in cocaine intake could still be noticed. After 2 consecutive daily sessions without TsA injection, rats still self-injected significantly less cocaine than control animals, suggesting that some stable mechanism was instated in response to repeated transitory HDAC inhibition (data not shown).

After 7 sessions of FR1 schedule, rats were submitted to daily sessions under a PR schedule of reinforcement, in which NPs required for drug delivery followed an exponential ascending equation. Because the PR schedule required several selfadministration sessions for the rat to display a stable behavior, only the 3 last sessions (sessions 5-7) were taken into consideration for statistical analysis. Similarly to what we found under the FR1 schedule, no difference in the number of cocaine selfinfusions was observed between rats treated with saline or vehicle under the PR schedule (data not shown). Animals treated intravenously with 0.03 or $0.3 \mathrm{mg} / \mathrm{kg}$ TsA 30 min before the beginning of each session reached a statistically significant lower breaking point than vehicle-treated animals $\left(F_{(3,86)}=22.9 ; p<0.001\right)$. Figure $2 a$ shows the decrease in the breaking point, expressed as the number of self-injections reached within a session, during the three last sessions. During the last session, treatment with 0.3 $\mathrm{mg} / \mathrm{kg}$ TsA reduced the breaking point from 25 to 21 selfinjections. The reducing effect of TsA treatment on drug intake was more manifest when expressed as the number of NPs the rat achieved to obtain the last injection. During the last session, vehicle-treated animals performed 764 NPs into the active hole, whereas this number decreased drastically to 340 for rats treated with the higher TsA dose (data not shown) $\left(F_{(3,86)}=16.1 ; p<\right.$ $0.001)$. The effect can also be illustrated by expressing the total number of NPs performed by the rat during the entire session $\left(F_{(3,86)}=16.1 ; p<0.001\right)$. Although vehicle-treated rats performed 4090 total cumulated NPs, this number decreased to 1774 when considering rats treated with $0.3 \mathrm{mg} / \mathrm{kg}$ TsA (Fig. $2 b$ ). It is noteworthy that TsA had a similar effect on the last PR sessions when the previous FR1 sessions were conducted in the absence of TsA treatment (data not shown).

We compared the effects of TsA to those produced by phenylbutyrate and depudecin, two compounds that belong to distinct groups of HDAC inhibitors. Daily intravenous administration of phenylbutyrate $30 \mathrm{~min}$ before the self-administration sessions resulted in a dose-dependent decrease in cocaine intake under the FR1 schedule. Although the lower dose of $2 \mathrm{mg} / \mathrm{kg}$ produced no statistical significant decrease in cocaine intake, the higher dose of $20 \mathrm{mg} / \mathrm{kg}$ phenylbutyrate produced a reduction that was statistically significant from session 5 , for which a $26 \%$ reduction in 

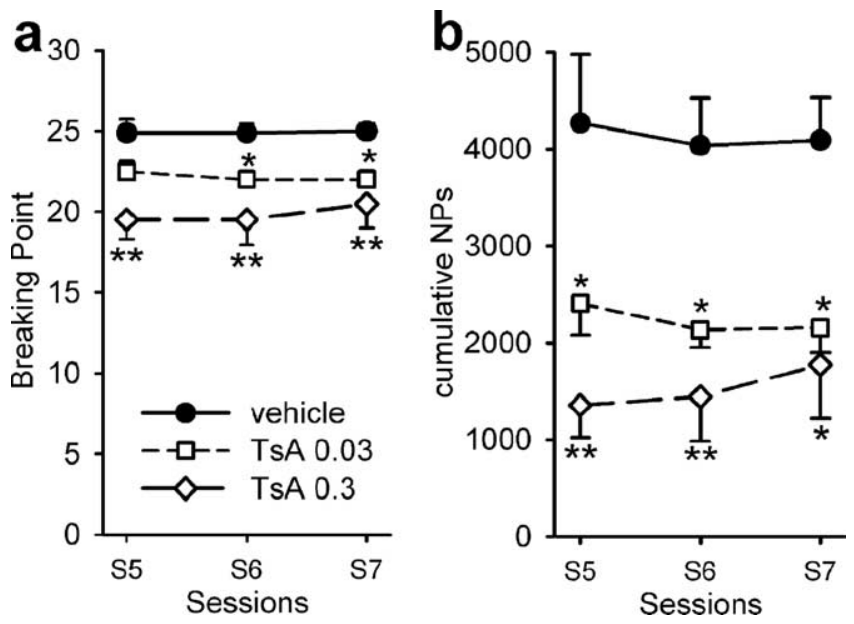

Figure 2. Effect of TsA on cocaine self-administration $(0.33 \mathrm{mg} / \mathrm{kg} /$ infusion $)$ under a PR schedule of reinforcement. PR sessions directly followed the FR sessions, and treatment of rats was as described in Figure 1.a, Breaking point achieved, expressed as number of self-injections; $\boldsymbol{b}$, cumulative number of NPs performed by the rat to reach the breaking point. Results are expressed as means \pm SEM. Vehicle-treated $(n=8), 0.03 \mathrm{mg} / \mathrm{kg}$ TsA-treated $(n=4)$, and 0.3 $\mathrm{mg} / \mathrm{kg}$ TsA-treated ( $n=7$ ) groups were studied. Results of the control saline-treated group $(n=10)$, which did not differ from those of the vehicle group are not shown but were taken into account for statistical analysis. ${ }^{*} p<0.05$ and ${ }^{* *} p<0.01$, comparison between vehicletreated and TsA-treated groups (ANOVA and Student-Newman-Keuls post hoc test).

self-injections was observed (supplemental Fig. S1, available at www.jneurosci.org as supplemental material). In contrast, the daily intracerebroventricular administration of 20 nmoles depucecin $30 \mathrm{~min}$ before the self-administration session did not significantly affect cocaine self-administration under the FR1 schedule, but it significantly decreased the breaking point reached during the PR sessions from 25 to 20 cocaine selfinfusions, similarly to TsA (supplemental Fig. S2, available at www.jneurosci.org as supplemental material).

\section{Effect of TsA on HDAC activity}

To ascertain that our protocol of intravenous injection of TsA was adequate for inhibiting brain HDAC activities, we measured total nuclear HDAC enzymatic activity in the FCx and NAc of rats treated with TsA. Figure 3 shows that daily injection of $0.3 \mathrm{mg} / \mathrm{kg}$ TsA for $4 \mathrm{~d}$ resulted in a significant decrease, by $\sim 40 \%$, of total HDAC activity $\left(\mathrm{FCx}: F_{(5,39)}=15.4, p<0.001\right.$; NAc: $F_{(5,39)}=5.9$, $p<0.01)$. A very similar decrease was observed in both structures when rats were treated for $3 \mathrm{~d}$ with an intraperitoneal injection of cocaine 30 min after the TsA treatment, the animals being killed 30 min after the TsA treatment on day 4. A comparable inhibition was noticed when the rats were submitted for $3 \mathrm{~d}$ to FR1 sessions of cocaine self-administration $30 \mathrm{~min}$ after each TsA treatment, the animals also being killed $30 \mathrm{~min}$ after the fourth TsA treatment.

\section{Effect of TsA on the cocaine-induced locomotor activity}

We next investigated whether the decrease in the motivation of the animals to self-administer cocaine resulted, at least partially, from an effect of TsA on their locomotor activity. No significant difference was observed in locomotor activity during a $2 \mathrm{~h}$ habituation period between groups of rats treated daily for $4 \mathrm{~d}$ with TsA or vehicle, the test being performed $30 \mathrm{~min}$ after the last injection (data not shown). When rats were injected with cocaine 30 min after the last TsA injection, an important hyperlocomotion was observed, which did not differ between the TsA- and the

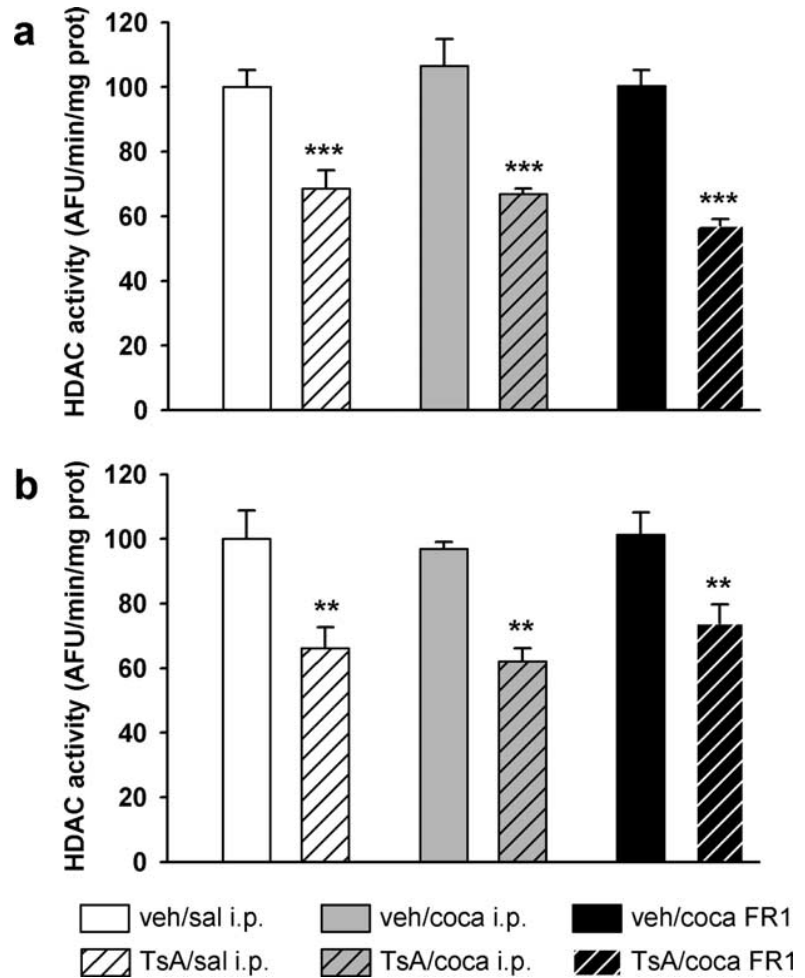

Figure 3. $\boldsymbol{a}, \boldsymbol{b}$, Effect of TsA on nuclear HDAC activity in the FCx $(\boldsymbol{a})$ and NAC $(\boldsymbol{b})$. TsA $(0.3$ $\mathrm{mg} / \mathrm{kg}$ ) or vehicle (veh) (10\% DMSO) were administered intravenously daily for $4 \mathrm{~d}$. Intraperitoneal injections $[0.9 \% \mathrm{NaCl}$ (sal) or $30 \mathrm{mg} / \mathrm{kg}$ cocaine (coca)] were injected daily during 3 consecutive days, $30 \mathrm{~min}$ after the TsA or vehicle injection. Other groups of rats were submitted to FR1 cocaine ( $0.33 \mathrm{mg} / \mathrm{kg} /$ injection) self-administration for 3 daily sessions beginning $30 \mathrm{~min}$ after the TsA injection. Rats were killed and the brain structures dissected 30 min after the fourth TsA injection (i.e., $24 \mathrm{~h}$ after the last cocaine administration). Total HDAC activity was assayed in the nuclear fraction prepared from each structure, using a fluorescent activity assay. Results are expressed as a percentage of saline-treated control, as means \pm SEM ( $n=6-9$ per group). ${ }^{* *} p<0.01$ and $^{* * *} p<0.001$, comparison to saline-treated group (ANOVA followed by Student-Newman-Keuls multiple comparisons test). AFU, Arbitrary fluorescence units; prot, protein.

vehicle-treated groups of rats (Fig. $4 a$ ). The intravenous injection protocol $(0.3 \mathrm{mg} / \mathrm{kg}$ TsA followed $30 \mathrm{~min}$ later by $1 \mathrm{mg} / \mathrm{kg}$ cocaine) was repeated daily for $5 \mathrm{~d}$. Control rats, as expected, responded to the successive cocaine treatments by a locomotor sensitization that was statistically significant from day 4 , whereas the TsA-treated animals did not show such an increased behavioral response during consecutive cocaine administration (Fig. $4 b$ ). When tested again at day 11 , the behavioral sensitization was stable in control animals and was still completely absent in TsAtreated rats $\left(F_{(1,83)}=28.6 ; p<0.001\right)$.

\section{Effect of TsA on sucrose self-administration}

We then checked whether the inhibition of HDAC activity in rat brain did affect their self-administration behavior toward the natural reinforcer sucrose. We used a very similar protocol than that used for cocaine administration, except that the rat had a sucrose solution to drink instead of receiving an intravenous drug infusion. During daily $2 \mathrm{~h}$ sessions of sucrose self-administration under the FR1 schedule, rats displayed a very stable level of sucrose intake during the 7 sessions. Daily intravenous administration of $0.3 \mathrm{mg} / \mathrm{kg}$ TsA $30 \mathrm{~min}$ before the beginning of the selfadministration session did not modify the sucrose intake behavior throughout the 7 sessions, when compared with rats that received vehicle (data not shown). 

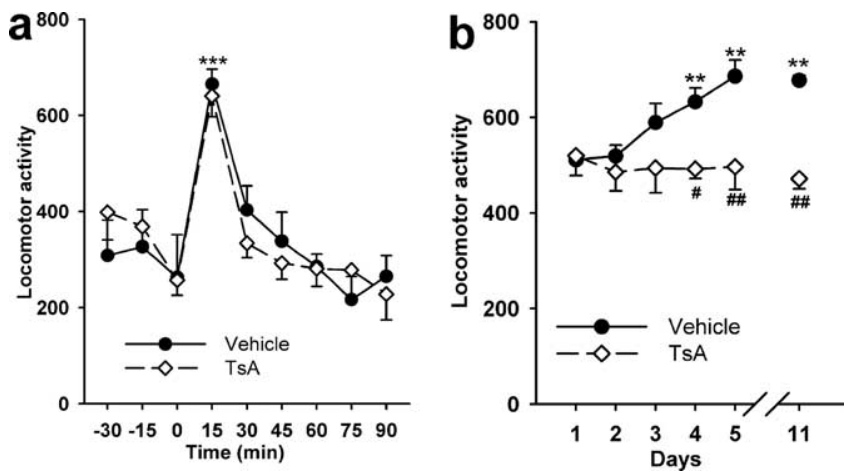

Figure 4. Effect of TsA on cocaine-induced locomotor activity. $\boldsymbol{a}$, Two groups of rats were given intravenous injections of TsA $(0.3 \mathrm{mg} / \mathrm{kg})$ or vehicle (10\% DMSO) daily for $4 \mathrm{~d}$. Thirty minutes after the fourth injection, both groups of rats were treated intravenously with $1 \mathrm{mg} / \mathrm{kg}$ cocaine (time 0 ). Locomotor activity was expressed over 15 min periods. $\boldsymbol{b}$, The intravenous injection protocol ( $0.3 \mathrm{mg} / \mathrm{kg}$ TsA followed $30 \mathrm{~min}$ later by $1 \mathrm{mg} / \mathrm{kg}$ cocaine) was repeated daily for 5 d. On day 11 , only cocaine was administered. Locomotor activity was expressed over $10 \mathrm{~min}$ periods. Each point represents the mean $\pm \operatorname{SEM}(n=6$, vehicle-treated group; $n=8$, TsAtreated group) of crossings. ${ }^{\#} p<0.05$; ${ }^{* *}$ or ${ }^{\# \#} p<0.01$ and ${ }^{* * *} p<0.001$. ${ }^{*}$ Significance when comparing each value to that obtained at time 0 in $\boldsymbol{a}$ or at day 1 in $\boldsymbol{b}$; ${ }^{\#}$ significance when comparing TsA group to vehicle group (ANOVA followed by Student-Newman-Keuls multiple comparisons test).
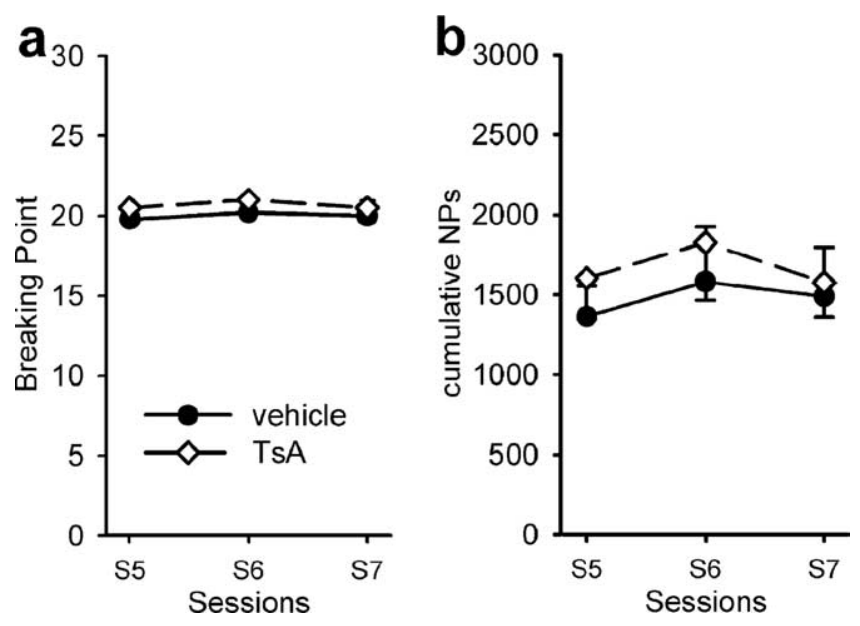

Figure 5. Effect of TsA on sucrose self-administration under a PR schedule of reinforcement. PR sessions directly followed the FR sessions, and treatment of rats was as described in legend to Figure 5. $\boldsymbol{a}$, Breaking point achieved, expressed as number of sucrose solutions delivered; $\boldsymbol{b}$, cumulative number of NPs performed by the rat to reach the breaking point. Results are expressed as means $\pm \operatorname{SEM}(n=5$ per group).

After 7 sessions of FR1 schedule, rats were submitted to daily sessions of sucrose intake under the PR schedule, which was identical to that used for estimating the motivation of rats for cocaine. Vehicle-treated control animals reached a breaking point of $\sim 20$ sucrose solutions delivered during each of the 3 last sessions (Fig. $5 a$ ) which was rather remarkable when compared with the 25 injections attained when cocaine was available. Surprisingly, the treatment with $0.3 \mathrm{mg} / \mathrm{kg}$ TsA was not found to modify the motivation of the animals for the natural reinforcer sucrose. Consequently, the total number of NPs performed by the rat during the entire session $(\sim 1500)$ was not different between the TsA- and the vehicle-treated group (Fig. $5 b$ ).

Because the self-administration experiments showed that HDAC inhibition did not decrease the motivation of rats for sucrose, whereas decreasing that for cocaine, we next investigated whether TsA would instead modify the hedonic aspect of the

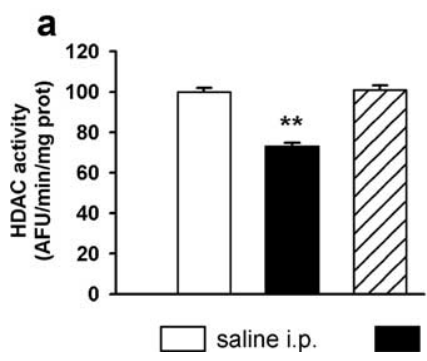

b

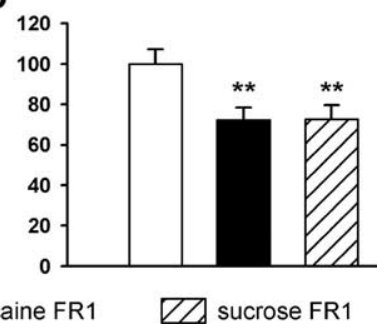

Figure 6. $\quad \boldsymbol{a}, \boldsymbol{b}$, Effect of cocaine or sucrose self-administration on nuclear HDAC activity in the $\mathrm{FCx}(\boldsymbol{a})$ and NAc $(\boldsymbol{b})$. Rats were submitted to 10 daily self-administration sessions for cocaine $(0.33 \mathrm{mg} / \mathrm{kg} /$ infusion, i.v.) or sucrose (delivery of $300 \mu \mathrm{l}$ of a $10 \%$ sucrose solution) under the FR1 schedule, or to daily injections of $0.9 \% \mathrm{NaCl}$ (saline). Two hours after the end of the last session or last injection, rats were killed and the brain structures dissected. Total HDAC activity was assayed in the nuclear fraction prepared from each structure, using a fluorescent activity assay. Results are expressed as a percentage of saline-treated control, as means \pm SEM $(n=7$ per group). ${ }^{* *} p<0.01$, comparison to saline-treated group (ANOVA followed by StudentNewman-Keuls multiple comparisons test). AFU, Arbitrary fluorescence units; prot, protein.

reward for sucrose. The sucrose preference test performed $30 \mathrm{~min}$ after the last of four daily injections with TsA indicated that the HDAC inhibitor did not decrease the volume of sucrose solution ingested. In effect, the TsA-treated rats drank $10.0 \pm 2.0 \mathrm{ml}$ of the sucrose solution during the test, compared with $9.7 \pm 1.7 \mathrm{ml}$ for the control vehicle-treated group. TsA was thus not found to affect the sucrose preference over water which was $81 \pm 3$ and $78 \pm 4 \%$ for control and TsA-treated groups, respectively.

Comparative effect of cocaine and sucrose on HDAC activity

Because the HDAC inhibitor regulated self-administration behavior for a drug of abuse and not for a natural reward, we searched whether the compounds would by themselves differentially modify brain HDAC enzymatic activity. Figure 6 shows that total HDAC activity from nuclear fractions was found to change when rats were submitted to 10 daily self-administration sessions for cocaine or sucrose (FCx: $F_{(2,20)}=32.9, p<0.001$; NAc: $F_{(2,20)}$ $=5.5, p<0.01)$. Interestingly, in the FCx, HDAC activity was significantly inhibited by the repeated cocaine intake but not by sucrose ingestion (Fig. 6a). However, total HDAC activity was similarly inhibited by both compounds in the NAc (Fig. 6b). Because the protocol used here differed markedly from that used in Figure 3 (namely in the number of self-administration sessions and in the delay after which animals were killed), results from Figures 3 and 6 are not comparable. The results suggest that the differential effect of TsA on cocaine and sucrose intake may be related with the different levels of HDAC enzymatic activities reached in discrete brain structures during treatment with the two reinforcers.

\section{Discussion}

The present study shows that injection of HDAC inhibitors to rats was sufficient to decrease the motivation of the animals to self-administer cocaine but not sucrose. Histone acetylation/ deacetylation mechanisms control modifications of chromatin structure that results in the regulation of gene transcription. It is generally thought that by removing acetyl groups from histones, HDAC enzymatic activity leads to gene repression through chromatin condensation. In mammalian cells, the HDACs are divided into four classes that depend on their structural homology to yeast deacetylases, expression pattern, and catalytic mechanisms (de Ruijter et al., 2003). The active site of class I and II HDACs is found within a conserved catalytic domain containing a zinc cat- 
ion, and most HDAC inhibitors function by displacing the cation. Inhibition of HDAC activity results in a general hyperacetylation of histones which is followed by the transcriptional activation of certain genes. We used three compounds that belong to distinct groups of inhibitors: TsA, a member of the hydroxamate group of HDAC inhibitors which displays an in vitro $\mathrm{IC}_{50}$ in the nanomolar range (Yoshida et al., 1990); depudecin, from the cyclic tetrapeptide group which has an affinity in the micromolar range (Kwon et al., 1998); and phenylbutyrate, from the group of aliphatic acids, displaying an affinity in the micromolar range (Butler and Bates, 2006). The inhibitors show no selectivity for particular members of class I or II HDACs (Butler and Bates, 2006).

To test the involvement of the chromatin architecture opening in drug reinforcement, we submitted rats to cocaine and sucrose operant self-administration paradigm. Because the procedure is based on an operant conditioning in which the animal itself regulates drug exposure, it has a good construct as well as appealing validity toward drug consumption in humans (Sanchis-Segura and Spanagel, 2006). Using the FR1 schedule, we found that TsA dose-dependently reduced the cocaine selfadministration behavior. Notably, the reduction persisted at least for two consecutive sessions without further TsA injection. The effect was of lesser magnitude when the higher cocaine dosage of $0.75 \mathrm{mg} / \mathrm{kg} /$ injection was infused (data not shown), suggesting that the HDAC inhibitor actually reduced the reinforcing properties of cocaine. We verified that the injection protocol we used for intravenous TsA delivery in the drug self-administration experiments produced indeed an inhibition of HDAC enzymatic activity in dopaminergic projection fields. For the highest TsA dosage, reduction in cocaine-self administration was statistically significant from the third session, suggesting that 3 daily sessions of TsA injection followed by cocaine infusion remodeled chromatin sufficiently to become behaviorally relevant by modulating the reinforcing properties of cocaine. This is in agreement with our previous data showing that passive administration of cocaine reduced histone $\mathrm{H} 3$ acetylation only after repeated injections (Cassel et al., 2006). It was also reported that chronic, but not acute, exposure to cocaine decreases HDAC5 function in the NAc, suggesting that proper balance of histone acetylation is a crucial factor in the salience of a given stimulus (Renthal et al., 2007).

Under the PR schedule of reinforcement, both TsA and depudecin were found to significantly reduce the breaking point, which is defined as the total number of cocaine infusions the rat self-administers within a given session. Although the repeated inhibition of HDACs greatly reduced the motivation of rats for cocaine, this was not the case when rats were allowed to selfadminister sucrose. The result shows that TsA treatment did not disturb the operant conditioning and that memory/learning mechanisms underlying the motivated behavior were not affected, as previously suggested by the elevated number of NPs (superior to $80 \%$ ) directed toward the active hole. Moreover, it is analogous to a recent report showing that repeated highfrequency stimulation of the FCx reduced both cocaine-seeking behavior and the motivation for its consumption but not sucrose reward-related behaviors (Levy et al., 2007). The differential effect between cocaine and the natural reward sucrose suggests that TsA did not diminish cocaine self-administration by a general impairment in the reward system. Although the two compounds probably overlap to a large extent, the neuronal circuitries of natural and drug-related rewards are not identical (Carelli, 2002). Perhaps, the underlying neurocircuitry of natural rewards that are fundamental for survival is less prone to long-term manipulations and to disruptions mediated by HDAC inhibitors. Incidentally, we found that HDAC activity in nuclear fractions prepared from the FCx was significantly inhibited by cocaine but not by sucrose self-administration. Although it is of course out of question to attribute modifications in a behavior as refined as drug self-administration to changes in a single enzymatic activity (even if this represents the algebraic sum of the activity of 11 distinct enzymes), it is nevertheless tempting to underline the correlation observed between the levels of HDAC enzymatic activities reached in the FCx during treatment with cocaine and sucrose on one side and the differential effect of HDAC inhibitors on the self-administration for the two compounds on the other side.

Our control experiments reveal that the TsA-induced decrease in the motivation for the drug is not attributable to a general malaise, or to a general motivational dysfunction because TsA had no adverse effect on novelty seeking or on cocaineinduced hyperlocomotion. The fact that the inhibition of HDAC activity by TsA had no negative effect on the sucrose preference test strongly suggests that the compound did not produce anhedonia, which would have explained a lack of motivation. In contrast, TsA was found to completely block the cocaine-induced behavioral sensitization. This phenomenon refers to a progressive increased locomotor response of rodents to intermittent administrations of the same dose of an addictive drug (Kalivas and Stewart, 1991). Given its context dependence and persistence, sensitization has been proposed as a central neural mechanism underlying addiction. An incentive-sensitization theory of addiction has been proposed that holds that, just as repeated drug administration sensitizes locomotor responses, it can also sensitize neural circuits that assign incentive salience (but not hedonic value) to drugs and drug-related cues (Robinson and Berridge, 1993). Sensitized incentive salience then produces intense wanting of drugs activated by drug-associated cues. In this line of thinking, our data reporting the blockade of locomotor sensitization by an HDAC inhibitor suggest that the inhibitor may also counteract the neural sensitization leading to drug dependence. This is in agreement with the decrease in the motivation of animals for cocaine we found using the self-administration PR schedule, when considering that (1) the neural sensitization theory implies a dysregulation of motivation; and (2) compulsive drug seeking derives, at least partially, from a drug-induced alteration of the motivation (Wise, 2004; Franken et al., 2005). Neurobiologically, glutamate plays an important role in the induction and expression of behavioral sensitization to stimulants (Vanderschuren and Kalivas, 2000). Based on the current results, decreased behavioral responses to cocaine are likely to result from a decreased glutamatergic neurotransmission controlled by genes, the expression of which derives from an epigenetic regulation of gene transcription.

Our results are in line with a recent report showing that chronic injection into the NAc of another HDAC inhibitor, valproic acid, attenuated amphetamine-induced locomotor activity in rats (Kim et al., 2008). In contrast to this and our present findings, a previous study showed that treatment of rats with the low-affinity HDAC inhibitor sodium butyrate enhanced the locomotor-activating effects of cocaine and that TsA administration to mice potentiated cocaine-induced place conditioning (Kumar et al., 2005). There are several possible explanations for the differences observed. There are methodological issues, such as species (rat versus mouse), circadian cycle (inverted in our study), length of drug administration (cocaine-induced locomo- 
tor effect was measured over a $2 \mathrm{~d}$ period only, whereas we ran the self-administration test over a 2 week period), type of drug administration (passively administered for locomotor activity and conditioned place preference, whereas in our study cocaine was self-administered), as well as type of analysis (locomotor activity and appetitive properties of cocaine, whereas we measured the reinforcing properties of cocaine and the motivation of animals to obtain the drug). Another possibility is that several histone modifications can take place in concert at the same histone tail, corresponding to various states of remodeled chromatin and therefore to the transcription of distinct genes (Cheung et al., 2000; Newton and Duman, 2006). Stability of the epigenetic events is another variable: although studies show that chromatin remodeling requires repeated treatments over several days (Cassel et al., 2006; Tsankova et al., 2006), chromatin can also be rapidly remodeled in response to neuronal stimulation (Chen et al., 2003).

Our data showing that epigenetic regulation of gene transcription taking place in postmitotic neurons is able to influence a motivated behavior put further emphasis on the emerging concept that epigenetic factors play a significant role in the mature working brain. Histone acetylation/deacetylation mechanisms play a key role in this process, and control differentially cocaineinduced reward and reward produced by a natural reinforcer. Some of the genes that are upregulated by the treatment with the HDAC inhibitors are valuable candidates for participating in the plasticity mechanisms occurring in response to drug intake and also for initiating the neurobiological alterations underlying the decrease in the motivation of rats to self-administer cocaine.

\section{References}

Arnold JM, Roberts DC (1997) A critique of fixed and progressive ratio schedules used to examine the neural substrates of drug reinforcement. Pharmacol Biochem Behav 57:441-447.

Bhat RV, Baraban JM (1993) Activation of transcription factor genes in striatum by cocaine: role of both serotonin and dopamine systems. J Pharmacol Exp Ther 267:496-505.

Butler R, Bates GP (2006) Histone deacetylase inhibitors as therapeutics for polyglutamine disorders. Nat Rev Neurosci 7:784-796.

Caine SB, Lintz R, Koob GF (1993) Intravenous drug self-administration techniques in animals. In: Behavioral neuroscience: a practical approach (Sahgal A, ed), pp 117-143. Oxford: Oxford UP.

Carelli RM (2002) Nucleus accumbens cell firing during goal-directed behaviors for cocaine vs. 'natural' reinforcement. Physiol Behav 76:379-387.

Cassel S, Carouge D, Gensburger C, Anglard P, Burgun C, Dietrich JB, Aunis D, Zwiller J (2006) Fluoxetine and cocaine induce the epigenetic factors MeCP2 and MBD1 in adult rat brain. Mol Pharmacol 70:487-492.

Chen WG, Chang Q, Lin Y, Meissner A, West AE, Griffith EC, Jaenisch R, Greenberg ME (2003) Derepression of BDNF transcription involves calcium-dependent phosphorylation of MeCP2. Science 302:885-889.

Cheung P, Allis CD, Sassone-Corsi P (2000) Signaling to chromatin through histone modifications. Cell 103:263-271.

Colvis CM, Pollock JD, Goodman RH, Impey S, Dunn J, Mandel G, Champagne FA, Mayford M, Korzus E, Kumar A, Renthal W, Theobald DE, Nestler EJ (2005) Epigenetic mechanisms and gene networks in the nervous system. J Neurosci 25:10379-10389.

de Ruijter AJ, van Gennip AH, Caron HN, Kemp S, van Kuilenburg AB (2003) Histone deacetylases (HDACs): characterization of the classical HDAC family. Biochem J 370:737-749.

Depoortere RY, Li DH, Lane JD, Emmett-Oglesby MW (1993) Parameters of self-administration of cocaine in rats under a progressive-ratio schedule. Pharmacol Biochem Behav 45:539-548.

Franken IH, Booij J, van den Brink W (2005) The role of dopamine in human addiction: from reward to motivated attention. Eur J Pharmacol 526:199-206.
Hope B, Kosofsky B, Hyman SE, Nestler EJ (1992) Regulation of immediate early gene expression and AP-1 binding in the rat nucleus accumbens by chronic cocaine. Proc Natl Acad Sci U S A 89:5764-5768.

Humblot N, Thiriet N, Gobaille S, Aunis D, Zwiller J (1998) The serotonergic system modulates the cocaine-induced expression of the immediate early genes egr-1 and c-fos in rat brain. Ann N Y Acad Sci 844:7-20.

Jenuwein T, Allis CD (2001) Translating the histone code. Science 293:1074-1080.

Jouvert P, Dietrich JB, Aunis D, Zwiller J (2002) Differential rat brain expression of EGR proteins and of the transcriptional corepressor NAB in response to acute or chronic cocaine administration. Neuromolecular Med 1:137-151.

Kalivas PW, Stewart J (1991) Dopamine transmission in the initiation and expression of drug- and stress-induced sensitization of motor activity. Brain Res Rev 16:223-244.

Kim WY, Kim S, Kim JH (2008) Chronic microinjection of valproic acid into the nucleus accumbens attenuates amphetamine-induced locomotor activity. Neurosci Lett 432:54-57.

Kumar A, Choi KH, Renthal W, Tsankova NM, Theobald DE, Truong HT, Russo SJ, Laplant Q, Sasaki TS, Whistler KN, Neve RL, Self DW, Nestler EJ (2005) Chromatin remodeling is a key mechanism underlying cocaineinduced plasticity in striatum. Neuron 48:303-314.

Kwon HJ, Owa T, Hassig CA, Shimada J, Schreiber SL (1998) Depudecin induces morphological reversion of transformed fibroblasts via the inhibition of histone deacetylase. Proc Natl Acad Sci U S A 95:3356-3361.

LeSage MG, Stafford D, Glowa JR (1999) Preclinical research on cocaine self-administration: environmental determinants and their interaction with pharmacological treatment. Neurosci Biobehav Rev 23:717-741.

Levy D, Shabat-Simon M, Shalev U, Barnea-Ygael N, Cooper A, Zangen A (2007) Repeated electrical stimulation of reward-related brain regions affects cocaine but not "natural" reinforcement. J Neurosci 27:14179-14189.

López-Rodas G, Brosch G, Georgieva EI, Sendra R, Franco L, Loidl P (1993) Histone deacetylase. A key enzyme for the binding of regulatory proteins to chromatin. FEBS Lett 317:175-180.

Moratalla R, Robertson HA, Graybiel AM (1992) Dynamic regulation of NGFI-A (zif268, egr1) gene expression in the striatum. J Neurosci 12:2609-2622.

Nestler EJ (2001) Molecular basis of long-term plasticity underlying addiction. Nat Rev Neurosci 2:119-128.

Newton SS, Duman RS (2006) Chromatin remodeling: a novel mechanism of psychotropic drug action. Mol Pharmacol 70:440-443.

Renthal W, Maze I, Krishnan V, Covington HE 3rd, Xiao G, Kumar A, Russo SJ, Graham A, Tsankova N, Kippin TE, Kerstetter KA, Neve RL, Haggarty SJ, McKinsey TA, Bassel-Duby R, Olson EN, Nestler EJ (2007) Histone deacetylase 5 epigenetically controls behavioral adaptations to chronic emotional stimuli. Neuron 56:517-529.

Richardson NR, Roberts DC (1996) Progressive ratio schedules in drug selfadministration studies in rats: a method to evaluate reinforcing efficacy. J Neurosci Methods 66:1-11.

Robinson TE, Berridge KC (1993) The neural basis of drug craving: an incentive-sensitization theory of addiction. Brain Res Rev 18:247-291.

Sanchis-Segura C, Spanagel R (2006) Behavioural assessment of drug reinforcement and addictive features in rodents: an overview. Addict Biol $11: 2-38$.

Tsankova NM, Berton O, Renthal W, Kumar A, Neve RL, Nestler EJ (2006) Sustained hippocampal chromatin regulation in a mouse model of depression and antidepressant action. Nat Neurosci 9:519-525.

Turner BM (2002) Cellular memory and the histone code. Cell 111:285-291.

Vanderschuren LJ, Kalivas PW (2000) Alterations in dopaminergic and glutamatergic transmission in the induction and expression of behavioral sensitization: a critical review of preclinical studies. Psychopharmacology (Berl) 151:99-120.

Wise RA (2004) Dopamine, learning and motivation. Nat Rev Neurosci 5:483-494.

Yoshida M, Kijima M, Akita M, Beppu T (1990) Potent and specific inhibition of mammalian histone deacetylase both in vivo and in vitro by trichostatin A. J Biol Chem 265:17174-17179. 